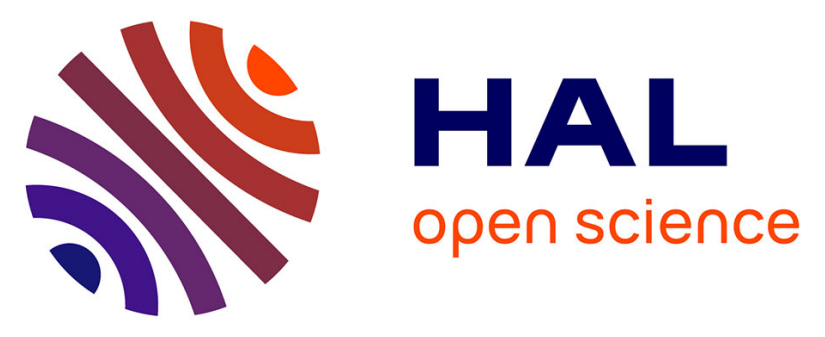

\title{
Sparsity-Based Algorithms for Blind Separation of Convolutive Mixtures with Application to EMG Signals
} Abdelwaheb Boudjellal, Karim Abed-Meraim, Abdeldjalil Aissa El Bey, Adel Belouchrani, Philippe Ravier

\section{- To cite this version:}

Abdelwaheb Boudjellal, Karim Abed-Meraim, Abdeldjalil Aissa El Bey, Adel Belouchrani, Philippe Ravier. Sparsity-Based Algorithms for Blind Separation of Convolutive Mixtures with Application to EMG Signals. IEEE Workshop on Statistical Signal Processing (SSP), Jun 2014, Gold Coast, Australia. Statistical Signal Processing Workshop (SSP), Australia, July 2014. hal-01002447

\section{HAL Id: hal-01002447 \\ https://hal.science/hal-01002447}

Submitted on 12 Jan 2015

HAL is a multi-disciplinary open access archive for the deposit and dissemination of scientific research documents, whether they are published or not. The documents may come from teaching and research institutions in France or abroad, or from public or private research centers.
L'archive ouverte pluridisciplinaire HAL, est destinée au dépôt et à la diffusion de documents scientifiques de niveau recherche, publiés ou non, émanant des établissements d'enseignement et de recherche français ou étrangers, des laboratoires publics ou privés. 


\section{SPARSITY-BASED ALGORITHMS FOR BLIND SEPARATION OF CONVOLUTIVE MIXTURES WITH APPLICATION TO EMG SIGNALS}
A. Boudjellal ${ }^{1}$
K. Abed-Meraim ${ }^{1}$
A. Aissa-El-Bey ${ }^{2}$
A. Belouchrani ${ }^{3}$
Ph. Ravier ${ }^{1}$

${ }^{1}$ Univ. of Orléans, PRISME Lab., France; ${ }^{2}$ Institut Telecom, Telecom Bretagne, France; ${ }^{3}$ Ecole Nationale Polytechnique, Algeria.

\begin{abstract}
In this paper we propose two iterative algorithms for the blind separation of convolutive mixtures of sparse signals. The first one, called Iterative Sparse Blind Separation (ISBS), minimizes a sparsity cost function using an approximate Newton technique. The second algorithm, referred to as Givens-based Sparse Blind Separation (GSBS) computes the separation matrix as a product of a whitening matrix and a unitary matrix estimated, via a Jacobi-like process, as the product of Givens rotations which minimize the sparsity cost function. The two sparsity based algorithms show significantly improved performance with respect to the time coherence based SOBI algorithm as illustrated by the simulation results and comparative study provided at the end of the paper.
\end{abstract}

Index Terms - Sparsity, Approximate Newton Technique, Givens Rotations, BSS of EMG Signals.

\section{INTRODUCTION}

A lot of signals present a sparse nature in the time domain or in another transformed domain. This property has been exploited in order to blindly separate a mixture of sources sharing this property. More precisely, recent works have demonstrated that by assuming that the sources can be represented sparsely in a given domain, Blind Source Separation (BSS) of a mixture of these sources is made possible by restoring this property $[5,6,8]$. Also, sources sparsity has been exploited for the nonnegative matrix factorization [5], channel deconvolution and sparse component analysis [7].

The main benefit of such sparse representation based approaches is the separation of a number of sources exceeding the number of observed mixtures (underdetermined BSS) [6] as for single-channel source separation [5]. Note Also that the use of sparsity can greatly improve the separation quality in overdetermined cases $[1,2]$.

Herein, we consider the blind separation of convolutive mixtures of sparse signals. Two different methods are introduced for minimizing a sparsity-based cost function. The first technique is an extension to the convolutive mixtures of the ISBS algorithm presented in [1, 2]. This algorithm has been used for the separation of instantaneous mixtures of audio signals based on their sparsity in the time domain [1] and the time-frequency domain [2]. The second technique proceeds to the minimization of the sparsity-based cost function using Givens rotations. The proposed algorithm has the advantage of fast convergence and good separation quality for a moderate computational cost. The first part of the paper is dedicated to the algorithms' development while the second part is devoted to the discussion of experimental results in the context of EMG (Electromyogram) source separation.

\section{PROBLEM FORMULATION}

In the context of multi-user MIMO systems, the convolutive mixtures $\mathbf{x}(t)$ of $d$ transmitted sources $\mathbf{s}(t)$ received through an $m$-antenna array is modeled as follows:

$$
\mathbf{x}(t)=\sum_{l=0}^{L-1} H_{l} \mathbf{s}(t-l)+\mathbf{n}(t) \stackrel{\text { def }}{=}[H(z)] \mathbf{s}(t)+\mathbf{n}(t)
$$

where $\mathbf{x}(t)=\left[x_{1}(t): x_{m}(t)\right]^{T}, \mathbf{s}(t)=\left[s_{1}(t): s_{d}(t)\right]^{T}$ is the vector of source signals assumed sparse in the time domain, $\mathbf{n}(t)=\left[n_{1}(t): n_{m}(t)\right]^{T}$ is an additive spatially and temporally white noise independent from $\mathbf{s}(t)$ and with covariance $\sigma^{2} \mathbf{I}_{m}$, and $H(z)=\sum_{l=0}^{L-1} H_{l} z^{-l}$ is the $m \times d$ mixing matrix filter assumed full column rank for all $z$.

Blind Source Separation aims to recover the unknown sources from observed mixtures, relying only on some assumptions on the statistical or structural properties of the original sources. This is equivalent to finding a $d \times m$ demixing matrix filter $W(z)=\sum_{r} W_{r} z^{-r}$ which output is the estimated source vector (up to a diagonal matrix filter [1]):

$$
\widehat{\mathbf{s}}(t)=[W(z)] \mathbf{x}(t)=\sum_{r} W_{r} \mathbf{x}(t-r)
$$

It has been shown in [3] that the convolutive mixture can be rewritten as an approximate instantaneous one by restructuring the model in (1) as follows:

$$
\overline{\mathbf{x}}(t)=\mathbf{H} \overline{\mathbf{s}}(t)+\overline{\mathbf{n}}(t)
$$

$\overline{\mathbf{x}}(t)=\left[\mathbf{x}(t)^{T}, \cdots, \mathbf{x}(t-K+1)^{T}\right]^{T}(K \geq d(L-1) /(m-d)$ is a window parameter), and $\overline{\mathbf{s}}(t)=\left[\mathbf{s}(t)^{T}, \cdots, \mathbf{s}(t-K-L+\right.$ 
$\left.1)^{T}\right]^{T}$. The $(m K) \times d(K+L-1)$ matrix $\mathbf{H}$ is given by:

$$
\mathbf{H}=\left[\begin{array}{ccccc}
H_{0} & \cdots & H_{L-1} & & 0 \\
& \ddots & & \ddots & \\
0 & & H_{0} & \cdots & H_{L-1}
\end{array}\right]
$$

By stacking $T$ samples of the restructured data vector $\overline{\mathbf{x}}(t)$ in one matrix $\mathbf{X}=[\overline{\mathbf{x}}(1): \overline{\mathbf{x}}(T)]$, the model (3) becomes:

$$
\mathbf{X}=\mathbf{H S}+\mathbf{N}
$$

where $\mathbf{S}=[\overline{\mathbf{s}}(1): \overline{\mathbf{s}}(T)]$. After the above transformation, recovering the unknown sources $\mathbf{S}$ from the observed data $\mathbf{X}$, is equivalent to finding a separation matrix $\mathbf{W}$ which output is the estimated source vector (up to inherent ambiguities), i.e.:

$$
\mathbf{Z}=\mathbf{W X}=\widehat{\mathbf{S}}
$$

Next, we present two methods for recovering the unknown sources by restoring their sparse nature which is measured by their $l_{p}$ norm where $0 \leq p<2$. The following cost function is used for quantifying the sparsity of the data:

$$
\begin{aligned}
\mathcal{G}_{p}(\mathbf{Z}) & =\frac{1}{m} \sum_{i=1}^{m}\left(\mathcal{J}_{p}\left(\mathbf{z}_{i}\right)\right)^{\frac{1}{p}} \\
\mathcal{J}_{p}\left(\mathbf{z}_{i}\right) & =\frac{1}{T} \sum_{j=1}^{T}\left|z_{i j}\right|^{p}
\end{aligned}
$$

where $z_{i j}$ is the $(i, j)^{t h}$ entry of the output data matrix $\mathbf{Z}$.

\section{ITERATIVE SPARSE BLIND SEPARATION ALGORITHM (ISBS)}

The Iterative Sparse Blind Separation (ISBS) algorithm [1] has been proposed for the separation of instantaneous mixtures of audio signals characterized by their sparse nature in the time domain. It has been extended to the convolutive mixtures [2] by reformulating the data model in the timefrequency domain. Herein, we propose to use the ISBS for the separation of convolutive mixtures by rearranging them into instantaneous mixtures as done in (3). The ISBS algorithm computes a separating matrix $\mathbf{W}$ as the minimizer of the sparsity criterion $\mathcal{G}_{p}$ and updated iteratively according to:

$$
\begin{aligned}
& \mathbf{Z}^{(k)}=\left(\mathbf{I}_{d}+\mu^{(k-1)}\right) \mathbf{Z}^{(k-1)} \\
& \mathbf{W}^{(k)}=\left(\mathbf{I}_{d}+\mu^{(k-1)}\right) \mathbf{W}^{(k-1)}
\end{aligned}
$$

The 'gradient' matrix $\mu^{(k)}$ is determined from a local linearization of $\mathcal{G}_{p}$. This optimization tool is an approximate Newton technique where the matrix $\mu^{(k)}$ can be very simply computed without any Hessian inversion as done in the Newton method. A first order Taylor expansion of (6) leads to:

$\mathcal{G}_{p}\left(\mathbf{Z}^{(k)}\right)=\mathcal{G}_{p}\left(\mathbf{Z}^{(k-1)}\right)+\frac{1}{m} \mathcal{R}\left\{\operatorname{Tr}\left(\mu^{(k-1)} Q^{(k-1)}\right)\right\}$

where $\mathcal{R}$ stands for the real part of a complex number, $Q_{q i}^{(k-1)}=\frac{p}{T} \sum_{j=1}^{T}\left|z_{i j}^{(k-1)}\right|^{p-1} e^{-\jmath \Phi_{i j}^{(k-1)}} z_{q j}^{(k-1)}$ with $\Phi_{i j}^{(k-1)}$ is the argument of the complex number $z_{i j}^{(k-1)}$. Using a gradient technique, the solution $\mu^{(k-1)}$ that minimizes (8) is:

$$
\mu^{(k-1)}=-\lambda Q^{(k-1) H} .
$$

$\lambda>0$ being a chosen step size parameter. Note that the ISBS algorithm is applied on the raw data without whitening stage. However, a whitening step represents a good starting point and prevents the algorithm from convergence to local minima.

\section{GIVENS-BASED SPARSE BLIND SEPARATION ALGORITHM (GSBS)}

We propose herein a new algorithm, referred to as GSBS based on Givens rotations for the minimization of the sparsity criterion. Its first step is a data whitening where the whitening matrix $\mathbf{B}$ is computed by using the classical eigendecomposition of the covariance matrix of the received signal $\mathbf{R}_{x}$ (i.e. the inverse square root of the data covariance matrix [4]). The whitened signal can then be written as:

$$
\mathbf{Y}=\mathbf{B X} \approx \mathbf{U}^{H} \mathbf{S}+\text { noise }
$$

where $\mathbf{U}=\mathbf{A}^{H} \mathbf{B}^{H}$ is a $d \times d$ unitary matrix. From (10), the separator can simply be expressed as: $\mathbf{W}=\mathbf{U B}$, which, in the noiseless case, results in $\mathbf{Z}=\mathbf{W X}=\mathbf{U B X}=\mathbf{S}$.

We recall that any $d \times d$ unitary matrix $\mathbf{U}$ can theoretically be decomposed into a product of $d(d-1) / 2$ elementary Givens rotations (a sweep of rotations) [4]. However, minimizing the sparsity criterion in (6) w.r.t. $\mathbf{U}$ when using exactly $d(d-1) / 2$ Givens rotations is a complex multidimensional optimization problem and, to the best of our knowledge, there is no simple solution or efficient algorithm to solve it. Hence, similarly to the Jacobi-like approaches, several sweeps are used in order to avoid this multi-dimensional optimization (i.e. only the parameters of the current rotation matrix are estimated at each step).

We propose herein an iterative Jacobi-like algorithm where the unitary matrix $\mathbf{U}$ is approximated by a finite product of elementary Givens rotations $\mathbf{G}^{(k)}(i, j, \theta, \alpha)$ such that:

$$
\mathbf{U}=\prod_{k=1}^{N_{s} d(d-1) / 2} \mathbf{G}^{(k)}(i, j, \theta, \alpha)
$$

where $N_{s}$ refers to the number of sweeps and $\mathbf{G}^{(k)}$ is the complex unitary Givens matrix with diagonal elements equal to one except for the two elements $g_{i i}=g_{j j}=\cos (\theta)$ and its off-diagonal elements are null except for the elements $g_{i j}=$ $-g_{j i}^{*}=e^{i \alpha} \sin (\theta)$. Hence, at each iteration $k$ the data matrix is updated according to $\mathbf{Z}^{(k)}=\mathbf{G}^{(k)} \mathbf{Z}^{(k-1)}$. The optimization of the sparsity criterion in (2) for $p=1$ w.r.t. the rotation parameters is equivalent to the minimization of:

$$
\mathcal{L}=\frac{1}{T} \sum_{t=1}^{T}\left(\sqrt{\mathbf{r}_{t}^{T} \mathbf{u}+\beta_{t}}+\sqrt{-\mathbf{r}_{t}^{T} \mathbf{u}+\beta_{t}}\right)+\lambda\left(\mathbf{u}^{T} \mathbf{u}-1\right)
$$


where $\lambda$ is a Lagrange multiplier, $\beta_{t}=\frac{1}{2}\left(\left|z_{i t}^{(k-1)}\right|^{2}+\left|z_{j t}^{(k-1)}\right|^{2}\right)$, $\mathbf{u}=[\cos (2 \theta), \sin (2 \theta) \cos (\alpha), \sin (2 \theta) \sin (\alpha)]^{T}$, and

$\mathbf{r}_{t}=\left[\frac{1}{2}\left(\left|z_{i t}^{(k-1)}\right|^{2}-\left|z_{j t}^{(k-1)}\right|^{2}\right), \mathcal{R}\left(z_{i t}^{(k-1)} z_{j t}^{(k-1) *}\right), \mathcal{I}\left(z_{i t}^{(k-1)} z_{j t}^{(k-1) *}\right)\right]^{T}$

Then, the desired solution satisfies:

$$
\frac{\partial \mathcal{L}}{\partial \mathbf{u}}=\frac{1}{T} \sum_{t=1} \frac{\mathbf{r}_{t}}{\sqrt{\mathbf{r}_{t}^{T} \mathbf{u}+\beta_{t}}}-\frac{\mathbf{r}_{t}}{\sqrt{-\mathbf{r}_{t}^{T} \mathbf{u}+\beta_{t}}}+2 \lambda \mathbf{u}=0
$$

Considering the approximation $\frac{1}{\sqrt{1+x}} \approx 1-\frac{1}{2} x+o\left(x^{2}\right)$, the zeroing of the derivative in (13) leads to:

$$
\left(\mathbf{V}-2 \lambda \mathbf{I}_{3}\right) \mathbf{u}=0
$$

with $\mathbf{V}=\sum_{t=1}^{T} \beta_{t}^{-3 / 2} \mathbf{r}_{t} \mathbf{r}_{t}^{T} / T$. Hence, the desired solution is an eigenvector of matrix $\mathbf{V}$. To find out which one should be considered, let's express the second order Taylor expansion of $\mathcal{L}$ for $\|\mathbf{u}\|=1$ (using $\sqrt{1+x}=1+\frac{1}{2} x-\frac{1}{8} x^{2}+o\left(x^{3}\right)$ ):

$$
\mathcal{L} \approx \frac{2}{T} \sum_{t=1}^{T} \sqrt{\beta_{t}}-\frac{1}{4} \mathbf{u}^{T} \mathbf{V u}
$$

and consequently the criterion is minimized when $\mathbf{u}$ is equal to the principal unit norm eigenvector of $\mathbf{V}$. Once $\mathbf{u}=\left[u_{1}, u_{2}, u_{3}\right]^{T}$ is computed, the couple of angles $(\theta, \alpha)$ minimizing equation (13) is given by:

$$
\cos (\theta)=\sqrt{\frac{u_{1}+1}{2}} \text { and } e^{i \alpha} \sin (\theta)=\frac{u_{2}+i u_{3}}{\sqrt{2\left(u_{1}+1\right)}}
$$

\section{APPLICATION TO EMG SIGNALS}

Skeletal Muscles are controlled by groups of motor units (MU). Each MU is made up of one Motor Neuron (MN) and a set of fibers that it innervates. The MN generates an impulse, called action potential (AP), which is transmitted to all the innervated muscle fibers of that particular MU [11]. The sum of all this electrical activity is referred to as a Motor Unit Action Potential (MUAP). Many motor units coordinate the contractions of a single muscle. The process of recording and evaluating the electrical activity produced by activated muscles is known as Electromyography (EMG) [10]. The EMG signal generated by the $j-$ th $\mathrm{MN}$ is modeled as follows:

$$
s_{j}(t)=M_{j}(t) * P_{j}(t)
$$

where $P_{j}(t)=\sum_{k \in \mathcal{N}} \delta\left(t-k T_{j}+\tau_{j, k}\right)$ is called the pulse nerve train (see [13] for details) and $M_{j}(t)$ is the waveform characterizing each MN. Each signal $s_{j}(t)$ will be transmitted to many muscle fibers across different channels (axons). The resulting electrical activity generated by the different received copies $s_{j}(t)$ constitute one MUAP. For enervating one muscle, many MUAPs are needed. The resulting electrical activity, observed via an $m$-antenna array, can be modeled as a convolutive mixture of the $d$-transmitted MUAPs [12]. Signal separation is needed here for a proper analysis of the MUAP components and sparsity is considered due to the sparse nature of the sources as illustrated in figure 2 .

\section{SIMULATION RESULTS}

To assess the performance of the two proposed algorithms, two simulation scenarios have been handled using (i) randomly generated sparse signals and (ii) EGM signals, respectively. In these simulation experiments, a $3 \times 5$ (i.e. $d=3$ and $m=5$ ) MIMO system is considered. The channel matrices $\left\{H_{l}, l=0, \cdots, L-1\right\}$ are generated randomly at each Monte Carlo run with i.i.d. Gaussian variables entries. The filter length $L$ is set to 3 and the window parameter $K$ is chosen equal to 3 . The algorithm's performance are compared to those of SOBI algorithm [4] through the Mean Rejection Level (MRL) [4] averaged over 500 Monte Carlo runs. The parameter $p$ is chosen equal to 1 for the ISBS and GSBS algorithms.

A convolutive mixture of random sparse signals is generated in the first simulation experiment. In figure 1, the plots represent the steady state MRL (obtained after 500 iterations) versus the sparsity degree of the randomly generated sparse signals. We note here that the higher is the sparsity degree the less sparse is the signal. We observe that SOBI is not suitable to that context while GSBS leads to the best separation quality. Also, severe performance degradation is observed when the degree of sparsity decreases.

In the second experiment, the three algorithms are applied to a convolutive mixture of EMG signals. Figure 2 represents an illustration example where we can see a sample of the original sources, an observed mixture and the separated sources using ISBS and GSBS, respectively.

The MRLs of the three algorithms versus the number of iterations are presented in figure 3. One can observe that, for EMG signals, the ISBS outperforms the GSBS in terms of convergence rate and separation quality.

\section{CONCLUSION}

In this paper, two new iterative algorithms have been introduced for the BSS of convolutive mixture of sparse signals. The two methods are based on the iterative minimization of sparsity-based cost function. The minimization of this latter is achieved via an approximate Newton method in the case of the ISBS algorithm and the use of Givens rotations in the case of GSBS algorithm. The simulation results illustrate their effectiveness as compared to SOBI algorithm which exploits the fact that the original sources are coherent over the time. For strongly sparse signals, the GSBS outperforms the ISBS while the latter leads to better results for real-life EMG source separation. 


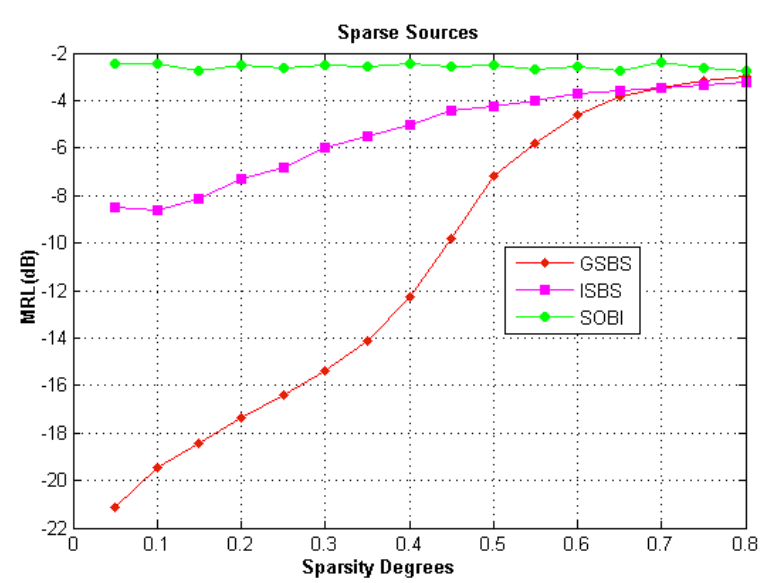

Fig. 1. MRL vs. Sparsity
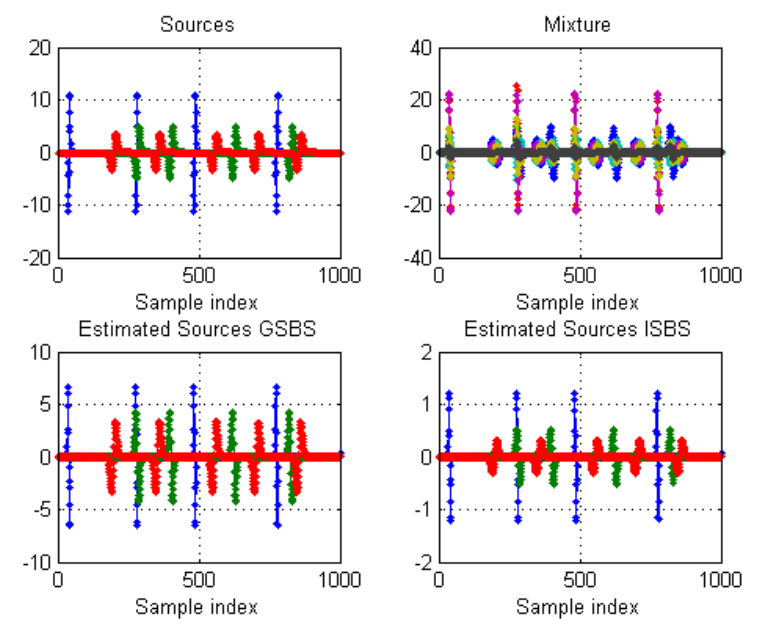

Fig. 2. EMG Sources.

\section{REFERENCES}

[1] A. Aissa-El-Bey, H. Bousbia-Salah, K. Abed-Meraim and Y. Grenier "Audio Source Separation Using Sparsity", Proc. IWAENC, Sep. 2006.

[2] A. Aissa-El-Bey, K. Abed-Meraim and Y. Grenier "Blind Audio Source Separation Using Sparsity based Criterion for Convolutive Mixture Case", Proc. ICA, Sep. 2007.

[3] Cedric Fevotte and Christian Doncarli, "A Unified Presentation of Blind Separation Methods for Convolutive Mixtures Using Block-Diagonalization”, Proc. ICA, April 2003.

[4] A. Belouchrani, K. Abed-Meraim, J.-F. Cardoso, and E. Moulines, "A blind source separation technique using second-order statistics”, IEEE Tr. on SP., Feb 1997.

[5] B. Gao, W. L. Woo, and S. S. Dlay, "Adaptive Sparsity NonNegative Matrix Factorization for Single-Channel Source Separation", IEEE J. of Selected Topics in SP, Sep. 2011

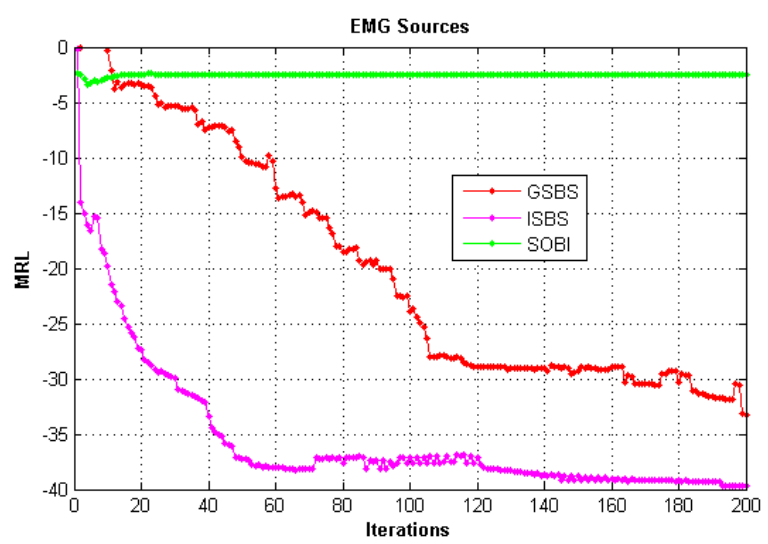

Fig. 3. MRL vs. Iterations: EMG Sources.

[6] Y. Li, S-I. Amari, A. Cichocki, D. W. C. Ho, and S. Xie, "Underdetermined Blind Source Separation Based on Sparse Representation", IEEE Tr. on SP, Feb. 2006.

[7] P. D. OGrady, B. A. Pearlmutter, and S. T. Rickard, "Survey of Sparse and Non-Sparse Methods in Source Separation", Int. Journal of Imaging Systems and Technology, 2005.

[8] R. Gribonval and S. Lesage, "A Survey of Sparse Component Analysis for Blind Source Separation : Principles, Perspectives, and New Challenges", Proc. ESANN, April 2006.

[9] J. Bobin, J. L.Starck, Y. Moudden, and M. J. Fadili, ”Blind Source Separation: the Sparsity Revolution", Chapter in Advances in Imaging and Electron Physics (Peter Hawkes, ed.), Academic Press, Elsevier, vol. 152, pp. 221-306, 2008.

[10] J. V. Basmajian and C. J. DeLuca, "Muscle Alive: Their Functions Revealed by Electromyography", Publisher: William and Wilkins; Editor: Baltimore MD, 1985.

[11] R. Merletti and P. A. Parker, "Electromyography Physiology, Engineering and Noninvasive Applications", John Wiley \& Sons, INC., IEEE Press, ISBN 0-471-67580-6, 2004.

[12] D. Farina, C. Févotte, Ch. Doncarli and R. Merletti, "Blind separation of Linear Instantaneous Mixtures of Nonstationary Surface Myoelectric Signals", IEEE Trans Biomed Eng, vol. 51, pp. 1555-1567, Sep. 2004.

[13] J. Roussel, M. Haritopoulos, Ph. Ravier, and O. Buttelli, "Cyclostationary Analysis of Electromyographic Signals", in Proc. EUSIPCO, 2013. 